\title{
SETS OF IDEMPOTENTS THAT GENERATE THE SEMIGROUP OF SINGULAR ENDOMORPHISMS OF A FINITE- DIMENSIONAL VECTOR SPACE
}

\author{
by R. J. H. DAWLINGS
}

(Received 1st October 1980)

If $\mathbf{M}$ is a mathematical system and End $\mathbf{M}$ is the set of singular endomorphisms of $\mathbf{M}$, then End $M$ forms a semigroup under composition of mappings. A number of papers have been written to determine the subsemigroup $S_{M}$ of End $M$ generated by the idempotents $E_{\mathbf{M}}$ of End $\mathbf{M}$ for different systems $M$. The first of these was by J. $M$. Howie [4]; here the case of $M$ being an unstructured set $X$ was considered. Howie showed that if $\mathrm{X}$ is finite, then End $\mathrm{X}=S_{\mathrm{X}}$.

Soon afterwards, J. A. Erdös [3] considered the case of $\mathbf{M}$ being a finite-dimensional vector space $\mathbf{V}$ over an arbitrary field $F$. Erdös showed that in this case also End $V=S_{\mathrm{v}}$. I have given two alternative proofs in [2]. J. B. Kim [6] has also given a proof of this result if the field $F$ is algebraically closed. The proofs given by Erdös and myself show that End $V$ is, in fact, generated by the subset $E$ of $E_{\mathrm{V}}$ consisting solely of the elements of $E_{\mathrm{v}}$ with one-dimensional null-space. It is easy to show that, except in the trivial case of $\mathbf{V}$ being a one-dimensional vector space, End $\mathbf{V}$ may be generated by a proper subset of $E$.

In this paper I determine conditions that are necessarily satisfied by a subset $E^{\prime}$ of $E$ if $E^{\prime}$ generates End $\mathbf{V}$. I then show, if the field $F$ is finite, that these conditions are also sufficient. From this, again if $F$ is finite, the minimum order of a generating set of idempotents is determined.

\section{Notation and preliminary results}

Definition 1.1. The semigroup of singular endomorphisms of an $n$-dimensional vector space $V$ over a field $F$ will be denoted by $\operatorname{Sing}_{n}$. Let $\alpha \in \operatorname{Sing}_{n}$. The range of $\alpha$ will be denoted by $\mathbf{R}_{\alpha}$ and the null-space of $\alpha$ by $\mathbf{N}_{\alpha}$. Elements of Sing ${ }_{n}$ will be written on the right of elements of the vector space $\mathbf{V}$.

Using this "right mapping" convention the following lemma is immediate:

Lemma 1.2. Let $\alpha, \beta \in \operatorname{Sing}_{n}$. Then:

(a) $\mathbf{N}_{\alpha} \subseteq \mathbf{N}_{\alpha \beta}$,

(b) $\mathbf{R}_{\alpha \beta} \subseteq \mathbf{R}_{\beta}$,

(c) $\alpha, \beta$ and $\alpha \beta$ all have the same rank if and only if $\mathbf{N}_{\alpha}=\mathbf{N}_{\alpha \beta}$ and $\mathbf{R}_{\alpha \beta}=\mathbf{R}_{\beta}$.

The following simple lemma will also be used. 
Lemma 1.3. ([1, Exercise 2.2.6.]) Let $\alpha, \beta \in$ Sing $_{n}$. Then:

(a) $\alpha \mathscr{L} \beta$ if and only if $\mathbf{R}_{\alpha}=\mathbf{R}_{\beta}$,

(b) $\alpha \mathscr{R} \beta$ if and only if $\mathbf{N}_{\alpha}=\mathbf{N}_{\beta}$,

(c) $\alpha \mathscr{D} \beta$ if and only if $\alpha$ and $\beta$ have the same rank,

(d) $\alpha \mathscr{J} \beta$ if and only if $\alpha \mathscr{D} \beta$.

Definition 1.4. The principal factor of $\operatorname{Sing}_{n}$ containing those elements of rank $n-1$ will be denoted by $P F_{n-1}^{0}$. The set of elements of Sing ${ }_{n}$ of rank $n-1$ will be denoted by $P F_{n-1}$. Thus $P F_{n-1}$ consists of the non-zero elements of $P F_{n-1}^{0}$.

The remainder of this section is devoted to introducing (and using) a new notation for the $\mathscr{H}$-classes of $P F_{n-1}^{0}$. This can quickly be adapted to serve as a new notation for elements of $E$ (the non-zero idempotents of $P F_{n-1}^{0}$ ).

Definition 1.5. Let $\xi, \chi$ be automorphisms of the field $F$ such that $\left(\chi \xi^{-1}\right)^{2}$ is the identity mapping. Let $\mathbf{a}=\left(a_{1}, a_{2}, a_{3}, \ldots, a_{n}\right)$ and $\mathbf{b}=\left(b_{1}, b_{2}, b_{3}, \ldots, b_{n}\right)$ be elements of $\mathbf{V}$. The $(\xi, \chi)$-stroke product (or simply stroke product) of $\mathbf{a}$ with $\mathbf{b}$ is denoted by $\langle\mathbf{a} \mid \mathbf{b}\rangle_{(\xi, x)}$ (or simply $\langle\mathbf{a} \mid \mathbf{b}\rangle)$ and defined by

$$
\langle\mathbf{a} \mid \mathbf{b}\rangle=\sum_{i=1}^{n}\left(a_{i} \xi\right)\left(b_{i} \chi\right)
$$

I shall regard $\xi$ and $\chi$ as fixed in advance and shall not make explicit reference to them in definitions and statements.

Definition 1.6. If $\mathbf{a}=\left(a_{1}, a_{2}, \ldots, a_{n}\right)$ and $\mathbf{b}=\left(b_{1}, b_{2}, \ldots, b_{n}\right)$ are elements of $\mathbf{V}$, we shall say that $\mathbf{a}$ and $\mathbf{b}$ are perpendicular if $\langle\mathbf{a} \mid \mathbf{b}\rangle=0$. It is simple to check that perpendicularity is a symmetric relation.

If $\mathbf{A}$ is a subset of $\mathbf{V}$, we shall define the perpendicular of $\mathbf{A}$ to be

$$
\mathbf{A}^{\perp}=\{\mathbf{x} \in \mathbf{V}:\langle\mathbf{x} \mid \mathbf{a}\rangle=\mathbf{0} \quad(\forall \mathbf{a} \in \mathbf{A})\}
$$

It should be noted that in general $\mathbf{A}$ and $\mathbf{A}^{\perp}$ are not disjoint. It should also be noted that $\mathbf{A}^{\perp}$ is a subspace of $\mathbf{V}$.

Using this definition of perpendicularity, the following lemma is simple to prove.

Lemma 1.7. Let $\mathbf{U}$ and $\mathbf{W}$ be subspaces of an $n$-dimensional vector space $\mathbf{V}$. Then:

(a) $\operatorname{dim} \mathbf{U}^{\perp}=\boldsymbol{n}-\operatorname{dim} \mathbf{U}$,

(b) $\left(\mathbf{U}^{\perp}\right)^{\perp}=\mathbf{U}^{\perp}$

(c) if $\mathbf{U} \subset \mathbf{W}$, then $\mathbf{W}^{\perp} \subset \mathbf{U}$.

Notation 1.8. Since every element in any particular $\mathscr{R}$-class of $P F_{n-1}^{0}$ has the same one-dimensional null-space we can label the $\mathscr{R}$-classes of $P F_{n-1}^{0}$ in the obvious way with an element of $\mathbf{V}$ that generates this one-dimensional subspace of $\mathbf{V}$. Similarly, the $\mathscr{L}$ classes of $P F_{n-1}^{0}$ could be labelled in the obvious way with $n-1$ elements of $V$ that generate the common range. But, since, if $\operatorname{dim} U=n-1$, we have (by Lemma 1.7) that $\operatorname{dim} U^{\perp}=1$, it follows that we can label the $\mathscr{L}$-classes of $P F_{n-1}^{0}$ in an obvious way with an element of $\mathbf{V}$ that generates the one-dimensional subspace of $\mathbf{V}$ perpendicular to the 
common range of the elements in that $\mathscr{L}$-class. Thus if $\alpha$ is a non-zero element of $P F_{n-1}^{0}$ such that $\mathbf{N}_{\alpha}=\langle\mathbf{n}\rangle$ and $\mathbf{R}_{\alpha}=\langle\mathbf{r}\rangle$ then we can label the $\mathscr{L}$-class containing $\alpha$ by $L_{\mathbf{r}}$, the $\mathscr{R}$-class containing $\alpha$ by $R_{\mathrm{n}}$ and the $\mathscr{H}$-class containing $\alpha$ by $H_{\mathrm{n}, \mathrm{r}}$. As $H_{\mathrm{n}, \mathrm{r}}$ is rather unwieldy this will in future be denoted by [n:r]. It is clear that [n:r] denotes exactly one $\mathscr{H}$-class for any choice of $\mathbf{n}$ and $\mathbf{r}$ in $\mathbf{V}$ (the fact that [n:r] represents at least one $\mathscr{H}$-class of $P F_{n-1}^{0}$ is a result of Lemma 1.3). It is also clear that for any non-zero scalars $\lambda$ and $\mu$ we have $[\mathbf{n}: \mathbf{r}]=[\lambda \mathbf{n}: \mu \mathbf{r}]$.

Having adopted this notation, it is then reasonable to introduce the following: If [n:r] is a group $\mathscr{H}$-class of $P F_{n-1}^{0}$ we shall denote the idempotent in [n:r] by (n:r). (n:r) is clearly unique since no $\mathscr{H}$-class contains more than one idempotent. With this notation there is a very simple way to tell if a particular $\mathscr{H}$-class of $P F_{n-1}^{0}$ contains an idempotent.

Lemma 1.9. [n:r] is a group $\mathscr{H}$-class if and only if $\langle\mathbf{n} \mid \mathbf{r}\rangle \neq 0$.

Proof. Suppose that [n:r] is a group $\mathscr{H}$-class. Then [n:r] contains the idempotent $\varepsilon$ $=(\mathbf{n}: \mathbf{r})$. Now $\mathbf{N}_{\varepsilon} \cap \mathbf{R}_{\varepsilon}=\{\mathbf{0}\}$ (for if $\mathbf{x} \in \mathbf{N}_{\varepsilon} \cap \mathbf{R}_{\varepsilon}$ then $\mathbf{x}=\mathbf{x} \varepsilon=\mathbf{0}$ ) and since $\mathbf{n} \in \mathbf{N}_{\varepsilon}$ and $\mathbf{n} \neq \mathbf{0}$ we have $\mathbf{n} \notin \mathbf{R}_{\varepsilon}=\left(\mathbf{R}_{\varepsilon}^{\perp}\right)^{\perp}$. But, since $\mathbf{r} \in \mathbf{R}_{\varepsilon}$ and $\mathbf{R}_{\varepsilon}^{\perp}$ is one-dimensional, we have $\langle\mathbf{n} \mid \mathbf{r}\rangle \neq 0$.

Conversely, suppose $\langle\mathbf{n} \mid \mathbf{r}\rangle \neq 0$. Now, there exists an element $\alpha \in P F_{n-1}^{0}$ such that $\mathbf{N}_{a}$ $=\langle\mathbf{n}\rangle$ and $\mathbf{R}_{\alpha}^{\perp}=\langle\mathbf{r}\rangle$. Since $\langle\mathbf{n} \mid \mathbf{r}\rangle \neq \mathbf{0}$, we have $\lambda \mathbf{n} \notin\left(\mathbf{R}_{\alpha}^{\perp}\right)^{\perp}=\mathbf{R}_{\alpha}$ for any non-zero scalar $\lambda$ in $F$, i.e. $\mathbf{R}_{\alpha} \cap \mathbf{N}_{\alpha}=\{\mathbf{0}\}$. Let $\mathbf{x} \in \mathbf{N}_{\alpha^{2}}$. Then $\mathbf{x} \alpha \in \mathbf{R}_{\alpha} \cap \mathbf{N}_{\alpha}$. Thus $\mathbf{x} \alpha=\mathbf{0}$ and so $\mathbf{x} \in \mathbf{N}_{\alpha}$. Consequently $\mathbf{N}_{\alpha^{2}} \subseteq \mathbf{N}_{\alpha^{2}}$. But $\mathbf{N}_{\alpha} \subseteq \mathbf{N}_{\alpha^{2}}$ and so $\mathbf{N}_{\alpha}=\mathbf{N}_{\alpha^{2}}$. Thus $\alpha \mathscr{R} \alpha^{2}$. Also, since $\operatorname{dim} \mathbf{N}_{\alpha}$ $=\operatorname{dim} \mathbf{N}_{\alpha^{2}}$, we have $\operatorname{dim} \mathbf{R}_{\alpha}=\operatorname{dim} \mathbf{R}_{\alpha^{2}}$. But $\mathbf{R}_{\alpha^{2}} \subseteq \mathbf{R}_{\alpha}$ and so $\mathbf{R}_{\alpha}=\mathbf{R}_{\alpha^{2}}$. Thus $\alpha \mathscr{L} \alpha^{2}$. Hence $\alpha \mathscr{H} \alpha^{2}$. So (by [5, Theorem II.2.5.]) $H_{\alpha}$ is a group and so contains an idempotent. Since $H_{\alpha}=[\mathbf{n}: \mathbf{r}]$, the result is proved.

Lemma 1.10. Let $\alpha$ and $\beta$ be elements of $P F_{n-1}^{0}$ in $[\mathbf{n}: \mathbf{r}]$ and $[\mathrm{m}: \mathrm{s}]$ respectively. Then $\alpha \beta \neq 0$ if and only if $\langle\mathbf{m} \mid \mathbf{r}\rangle \neq 0$.

Proof. Suppose first that $\alpha \beta \neq 0$. Then $\alpha \beta, \alpha$ and $\beta$ all have the same rank. So, by Lemma 1.2 and Lemma 1.3, $\alpha \beta \in R_{\alpha} \cap L_{\beta}$. By [1, Theorem 2.17.], $R_{\beta} \cap L_{\alpha}$ contains an idempotent, i.e. $\langle\mathbf{m} \mid \mathbf{r}\rangle \neq 0$.

Now suppose that $\langle\mathbf{m} \mid \mathbf{r}\rangle \neq 0$. Then $R_{\beta} \cap L_{\alpha}$ contains an idempotent. So, again by [1, Theorem 2.17.], $\alpha \beta \in R_{\alpha} \cap L_{\beta}$. Thus $\alpha \beta$ has rank $n-1$, and so $\mu \beta=0$.

\section{The necessary conditions}

In this section necessary conditions are found for a subset $E^{\prime}$ of $E$ to generate $\operatorname{Sing}_{n}$. Throughout this section there are no restrictions on the field $F$ over which the vector space $\mathbf{V}$ is defined.

Definition 2.1. Let $E^{\prime}$ be a subset of $E$. We shall say that $E^{\prime}$ covers [sparsely covers] $P F_{n-1}^{0}$ if $E^{\prime}$ has non-empty intersection with [intersects in exactly one element] each non-zero $\mathscr{L}$-class and each non-zero $\mathscr{R}$-class of $P F_{n-1}^{0}$. We shall also say that $E^{\prime}$ covers $P F_{n-1}$. 
Lemma 2.2. There exists a sparse covering set $E^{\prime}$ for $P F_{n-1}^{0}$.

Proof. The proof is by induction on the dimension $n$ of the vector space $V$. For clarity we shall denote the $m$-dimensional vector space by $\mathbf{V}_{m}$.

We now define a set of representatives $V_{m}^{\prime}$ of the one-dimensional subspaces of $V_{m}$. So, for all non-zero $\mathbf{x}$ in $\mathbf{V}_{m}$ there exists a unique $\mathbf{y}$ in $\mathbf{V}_{m}^{\prime}$ such that $\langle\mathbf{x}\rangle=\langle\mathbf{y}\rangle$. We shall denote by $L_{\mathrm{x}}^{m}\left[R_{\mathrm{x}}^{m}\right]$ the $\mathscr{L}$-class $\left[\mathscr{R}\right.$-class] of $P F_{m-1}^{0}$ containing those elements with range perpendicular to [null-space equal to] $\langle\mathbf{x}\rangle$.

Now suppose, as the induction hypothesis, that there exists a sparse covering set $E_{m}^{\prime}$ of $P F_{m-1}^{0}$. Then there exists exactly one element $\varepsilon$ in $L_{\mathbf{x}}^{m} \cap E_{m}^{\prime}$ for each $\mathbf{x} \in \mathbf{V}_{m}^{\prime}$. All the elements in $\mathbf{R}_{\varepsilon}$ have the same null-space, generated by a particular element of $V_{m}^{\prime}$. If we denote this element by $\mathbf{y}(\mathbf{x})$, we have, in fact, defined a mapping $V_{m}^{\prime} \rightarrow V_{m}^{\prime}$ by $\mathbf{x} \mapsto \mathbf{y}(\mathbf{x})$. This mapping is characterised by $L_{\mathrm{x}}^{m} \cap R_{\mathrm{y}(\mathrm{x})}^{m} \cap E_{m}^{\prime}$ being non-empty.

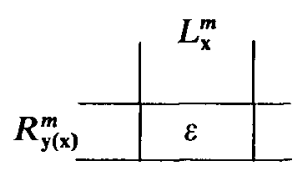

This mapping is clearly a bijection. Notice that there exists an idempotent, namely $\varepsilon$, with null-space $\langle\mathbf{y}(\mathbf{x})\rangle$ and range $\langle\mathbf{x}\rangle^{\perp}$. Thus $[\mathbf{y}(\mathbf{x}): \mathbf{x}]$ is a group $\mathscr{H}$-class, and so $\langle\mathbf{y}(\mathbf{x}) \mid \mathbf{x}\rangle \neq 0$.

If $\mathbf{x}=\left(x_{1}, x_{2}, \ldots, x_{m}\right)$ is an element of $\mathbf{V}_{m}^{\prime}$ and $a \in F$, then denote by $(\mathbf{x}, a)$ the element of $\mathbf{V}_{m+1}^{\prime}$ that generates the space $\left\langle\left(x_{1}, x_{2}, \ldots, x_{m}, a\right)\right\rangle$. We shall denote by $(0,1)$ the element of $\mathbf{V}_{m+1}^{\prime}$ that generates the space $\langle(0,0, \ldots, 0,1)\rangle$. Clearly, these are all distinct, and every element of $\mathbf{V}_{m+1}^{\prime}$ may be denoted in this way. Notice that if $\mathbf{y}=\left(y_{1}, y_{2}, \ldots, y_{m+1}\right)$, then for some $\mathbf{x} \in \mathbf{V}_{m}^{\prime} \cup\{\mathbf{0}\}$ and some $\lambda, a \in F$ we have $\left(y_{1}, y_{2}, \ldots, y_{m}\right)=\lambda \mathbf{x}$ and $y_{m+1}=\lambda a$.

We shall now set up a bijection $\overline{\mathbf{y}}: V_{m+1}^{\prime} \rightarrow V_{m+1}^{\prime}$ such that $L_{(\mathbf{x}, a)}^{m+1} \cap R_{\mathrm{y}(\mathbf{x}, a)}^{\mathrm{m}+1}$ is a group $\mathscr{H}$ class of $P F_{m}^{0}$ for all $\mathbf{x}$ in $V_{m}^{\prime}$ and all $a$ in $F$, and also such that $L_{(0,1)}^{m+1} n R_{(0,1)}^{m+1}$ is a group $\mathscr{H}$ class of $P F_{m}^{0}$. We shall construct $\bar{y}$ so that for $\mathbf{x}$ in $V_{m}^{\prime}$ and $a$ in $F$ we have $\bar{y}(\mathbf{x}, a)$ $=(\mathbf{y}(\mathbf{x}), z)$ for some $z$ in $F$. We need to have $\langle\tilde{\mathbf{y}}(\mathbf{x}, a) \mid(\mathbf{x}, a)\rangle \neq 0$, and so we must have $\langle\mathbf{y}(\mathbf{x}) \mid \mathbf{x}\rangle+(z \xi)(a \chi) \neq 0$. Now, by the definition of $\mathbf{y}(\mathbf{x})$, we know that $\langle\mathbf{y}(\mathbf{x}) \mid \mathbf{x}\rangle \neq 0$. Thus, if $a \neq 0$, we need $z \xi \neq-\langle\mathbf{y}(\mathbf{x}) \mid \mathbf{x}\rangle /(a \chi)$ and, if $a=0, z$ may take any value we choose. We know that $F$ contains the elements 0 and 1 . Thus if $a \neq 0$, we may put $z \xi=$ $1-\langle\mathbf{y}(\mathbf{x}) \mid \mathbf{x}\rangle /(a \chi)$. The only value that this may not take is 1 since $\langle\mathbf{y}(\mathbf{x}) \mid \mathbf{x}\rangle \neq 0$. So if $a=0$ we shall set $z=1$. Thus

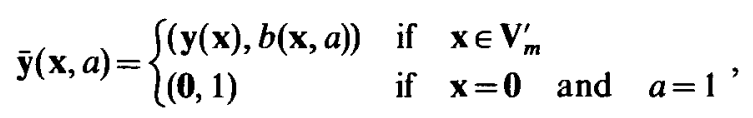

where

$$
b(\mathbf{x}, a)=\left\{\begin{array}{ll}
{[1-\langle\mathbf{y}(\mathbf{x}) \mid \mathbf{x}\rangle /(a \chi)] \xi^{-1}} & \text { if } \quad a \neq 0 \\
1 \xi^{-1}=1 & \text { if } a=0
\end{array} .\right.
$$

It is easy to check that $\overline{\mathbf{y}}$ is a bijection. 
From the definition of $\overline{\mathbf{y}}$ we have that for all $(\mathbf{x}, a)$ in $\mathbf{V}_{m+1}^{\prime},\langle\overline{\mathbf{y}}(\mathbf{x}, a) \mid(\mathbf{x}, a)\rangle \neq 0$. Thus $L_{(x, a)}^{m+1} \cap R_{y(x, a)}^{m}$ contains an idempotent. Hence the set $E_{m+1}^{\prime}=\left\{(\bar{y}(\mathbf{x}, a):(\mathbf{x}, a)):(\mathbf{x}, a) \in \mathbf{V}_{m+1}^{\prime}\right\}$ is a sparse cover for $P F_{m}^{0}$.

It remains to show that we may anchor the induction at $m=2$. Since every onedimensional subspace of $V_{2}$ may be generated by a vector of the form $(1, a)$ or by the vector $(0,1)$, it is easy to see that the set

$$
\left\{\left(\left(1,\left(1-(a \chi)^{-1}\right) \xi^{-1}\right):(1, a)\right): a \in F \backslash\{0\}\right\} \cup[((1,1):(1,0)),((0,1):(0,1))\}
$$

forms a sparse cover for $P F_{1}^{0}$.

Definition 2.3. Let $E^{\prime}$ be a subset of $E$ and $\phi, \gamma \in E^{\prime}$. Then the relation $\pi\left(E^{\prime}\right)$ is defined by: $(\phi, \gamma) \in \pi\left(E^{\prime}\right)$ if there exist elements $\varepsilon_{1}, \varepsilon_{2}, \ldots, \varepsilon_{p}$ in $E^{\prime}$ such that $\phi \varepsilon_{1} \varepsilon_{2} \ldots \varepsilon_{p} \gamma \in P F_{n-1}$.

Lemma 2.4. If $E^{\prime}$ is a subset of $E$ and $E^{\prime}$ generates $\operatorname{Sing}_{n}$, then $E^{\prime}$ covers $P F_{n-1}$ and $\pi\left(E^{\prime}\right)$ is the universal relation on $E^{\prime}$.

Proof. Let $\beta$ be any element of $P F_{n-1}$. Since $E^{\prime}$ generates $\operatorname{Sing}_{n}$, it certainly generates $P F_{n}{ }_{1}$. Thus there exist elements $\varepsilon_{1}, \varepsilon_{2}, \ldots, \varepsilon_{p}$ in $E^{\prime}$ such that $\beta=\varepsilon_{1} \varepsilon_{2} \ldots \varepsilon_{p}$. Now, since rank $\beta=\operatorname{rank} \varepsilon_{i}(i=1,2, \ldots, p)$, we have that $\mathbf{N}_{\beta}=\mathbf{N}_{\varepsilon_{1}}$ and $\mathbf{R}_{\beta}=\mathbf{R}_{\varepsilon_{p}}$. Thus $\beta \mathscr{R} \varepsilon_{1}$ and $\beta \%^{\prime} \ddot{c}_{p}$. Hence both $R_{\beta} \cap E^{\prime}$ and $L_{\beta} \cap E^{\prime}$ are non-empty. Since $\beta$ was chosen arbitrarily, it follows that $E^{\prime}$ covers $P F_{n-1}$.

Now let $\phi, \gamma \in E^{\prime}$, and let $\alpha \in R_{\phi} \cap L_{\gamma}$. Since $E^{\prime}$ generates $\alpha$ we have that $\alpha=\varepsilon_{1} \varepsilon_{2} \ldots \varepsilon_{p}$ for some $\varepsilon_{1}, \varepsilon_{2}, \ldots, \varepsilon_{p}$ in $E^{\prime}$. But $\varepsilon_{1} \mathscr{R} \alpha$ and $\varepsilon_{p} \mathscr{L} \alpha$. Thus $\phi \mathscr{R} \varepsilon_{1}$ and $\gamma \mathscr{L} \varepsilon_{p}$. Hence $\phi \varepsilon_{1}=\varepsilon_{1}$ and $\varepsilon_{p} \gamma=\varepsilon_{p}$. So $\alpha=\phi \varepsilon_{1} \varepsilon_{2} \ldots \varepsilon_{p} \gamma$, i.e. $\phi \varepsilon_{1} \varepsilon_{2} \ldots \varepsilon_{p} \gamma \in P F_{n-1}$. Since $\varepsilon_{1}, \varepsilon_{2}, \ldots, \varepsilon_{p} \in E^{\prime}$, we have that $(\phi, \gamma) \in \pi\left(E^{\prime}\right)$. Since $\phi$ and $\gamma$ were chosen arbitrarily, it follows that $\pi\left(E^{\prime}\right)$ is the universal relation on $E^{\prime}$.

\section{Sufficient conditions and minimum order generating sets when $F$ is a finite field}

Throughout this section we shall take $F$ to be the finite field with $q$ elements.

Theorem 3.1. Let $\mathbf{V}$ be an n-dimensional vector space over a finite field $F$. Let Sing be the semigroup of singular endomorphisms of $\mathrm{V}$ and let $P F_{n-1}$ be the set of elements in Sing $_{n}$ with rank $n-1$. Let $E^{\prime}$ be a subset of the idempotents of $P F_{n-1}$. Then $E^{\prime}$ generates Sing $_{n}$ if and only if $\pi\left(E^{\prime}\right)$ is the universal relation on $E^{\prime}$ and $E^{\prime}$ covers $P F_{n-1}$.

Proof. We have already shown that if $E^{\prime}$ generates $\operatorname{Sing}_{n}$ then $\pi\left(E^{\prime}\right)$ is universal on $E^{\prime}$ and $E^{\prime}$ covers $P F_{n-1}$.

To show the converse it will suffice to show that $E^{\prime}$ generates $E$, the set of all idempotents in $P F_{n-1}$, for (by [3]) we have that $E$ generates $S_{i n g}$.

Let $x \in E$. Since $E^{\prime}$ covers $P F_{n-1}$, there exist $\phi, \gamma$ in $E^{\prime}$ such that $\phi \mathscr{R} \varepsilon$ and $\gamma \mathscr{L} \varepsilon$. Since $\pi\left(E^{\prime}\right)$ is universal on $E^{\prime}$, we have that $(\phi, \gamma) \in \pi\left(E^{\prime}\right)$. Hence there exist $\varepsilon_{1}, \varepsilon_{2}, \ldots, \varepsilon_{p}$ in $E^{\prime}$ such that $\alpha=\phi \varepsilon_{1} \varepsilon_{2} \ldots \varepsilon_{p} \gamma$ has rank $n-1$. Now, $\mathbf{N}_{\alpha}=\mathbf{N}_{\phi}$ and $\mathbf{R}_{\alpha}=\mathbf{R}_{\gamma}$. Thus $\alpha \mathscr{R} \phi$ and $\alpha \mathscr{L} \gamma$. Hence $\alpha \mathscr{R} \varepsilon$ and $\alpha \mathscr{L} \varepsilon$, i.e. $\alpha \mathscr{H} \varepsilon$. Now, since $F$ is finite, $\operatorname{Sing}_{n}$ is finite and so 
certainly $H_{\varepsilon}$ is finite. So $\alpha$ belongs to a finite group. Thus, for some integer $k \geqq 1, \alpha^{k}$ is the identity of that group, i.e. $\alpha^{k}=\varepsilon$. Since $\alpha$ is a product of elements of $E^{\prime}$, we have that $E^{\prime}$ generates $\varepsilon$. But this holds for all elements of $E$ and so $E^{\prime}$ generates $E$ as required.

The next three lemmas will be used in the proof of Theorem 3.5.

Lemma 3.2. If $|F|=q$, then the number of non-zero $\mathscr{L}$-classes $\left[\mathscr{R}\right.$-classes] in $P F_{n-1}^{0}$ is $\left(q^{n}-1\right) /(q-1)$.

Proof. By Lemma 2.2, we know that there is a bijection between the elements of a sparse cover of $P F_{n-1}^{0}$ and the $\mathscr{L}$-classes [R्R-classes] of $P F_{n-1}^{0}$. Thus there is a bijection between the $\mathscr{L}$-classes and $\mathscr{R}$-classes of $P F_{n-1}^{0}$. Since $F$ is finite it follows that $P F_{n-1}^{0}$ is finite and so there are only finitely many $\mathscr{L}$-classes [ $\mathscr{R}$-classes] in $P F_{n-1}^{0}$. Consequently there are the same number of $\mathscr{L}$-classes as $\mathscr{R}$-classes in $P F_{n-1}^{0}$.

From the comments of Notation 1.8, we know that there is a bijection between the one-dimensional subspaces of $\mathrm{V}$ and the non-zero $\mathscr{L}$-classes of $P F_{n-1}^{0}$. Now, the number of non-zero vectors in $\mathbf{V}$ is $q^{n}-1$. However, for each $\mathbf{x}$ in $\mathbf{V}$ and for all non-zero scalars $\lambda$ in $F$, we have $\langle\mathbf{x}\rangle=\langle\lambda \mathbf{x}\rangle$. Hence there are $\left(q^{n}-1\right) /(q-1)$ one-dimensional subspaces in V.

Lemma 3.3. If $|F|=q$, then the number of idempotents in any non-zero $\mathscr{L}$-class $[\mathscr{R}$ class] of $P F_{n-1}^{0}$ is $q^{n-1}$.

Proof. The number of idempotents in a given $\mathscr{L}$-class $L$ is the number of $\mathscr{R}$-classes containing an idempotent in $L$, i.e. the number of $\mathscr{R}$-classes which intersect $L$ in a group. If the elements in $L$ have range $\langle\mathbf{r}\rangle$ then this is just $Q=|\{\langle\mathbf{n}\rangle:\langle\mathbf{n} \mid \mathbf{r}\rangle \neq 0\}|$. Since the number of one-dimensional subspaces of $V$ is $\left(q^{n}-1\right) /(q-1)$, we have that

$$
Q=\left(q^{n}-1\right) /(q-1)-|\langle\langle\mathbf{n}\rangle:\langle\mathbf{n} \mid \mathbf{r}\rangle=0\}| \text {. }
$$

But $\{\langle\mathbf{n}\rangle:\langle\mathbf{n} \mid \mathbf{r}\rangle=\mathbf{0}\}=\left\{\langle\mathbf{n}\rangle: \mathbf{n} \in\langle\mathbf{r}\rangle^{\perp}\right\}$. Since $\operatorname{dim}\langle\mathbf{r}\rangle^{\perp}=n-1$. we have that $\left|\left\{\langle\mathbf{n}\rangle: \mathbf{n} \in\langle\mathbf{r}\rangle^{1}\right\}\right|=\left(q^{n-1}-1\right) /(q-1)$. Thus $Q=\left(q^{n}-1\right) /(q-1)-\left(q^{n-1}-1\right) /(q-1)=q^{n-1}$ as required.

Lemma 3.4. If $\dot{F}$ is a finite field and $E^{\prime}$ is a sparse cover for $P F_{n-1}^{0}$, then $\pi\left(E^{\prime}\right)$ is the universal relation on $E^{\prime}$.

Proof. Let $\phi, \gamma$ be any two elements of $E^{\prime}$ and suppose that $\phi \pi\left(E^{\prime}\right) \cap \gamma\left[\pi\left(E^{\prime}\right)\right]^{-1}$ is empty. Since each $\mathscr{L}$-class of $P F_{n-1}^{0}$ contains $q^{n-1}$ idempotents and $E^{\prime}$ is a sparse cover of $P F_{n-1}^{0}$, we know that there are exactly $q^{n-1}$ elements $\varepsilon_{i}$ of $E^{\prime}$ such that $\phi \varepsilon_{i} \neq 0$ in $P F_{n-1}^{0}$ (by Lemma 1.9 and Lemma 1.10). Hence $\left|\phi \pi\left(E^{\prime}\right)\right| \geqq q^{n-1}$. Similarly, since each $\mathscr{R}-$ class of $P F_{n-1}^{0}$ contains $q^{n-1}$ idempotents, we have that there exist exactly $q^{n-1}$ elements $\varepsilon_{i}^{\prime}$ of $E^{\prime}$ such that $\varepsilon_{i}^{\prime} \gamma \neq 0$ in $P F_{n-1}^{0}$. Thus $\left|\gamma\left[\pi\left(E^{\prime}\right)\right]^{-1}\right| \geqq q^{n-1}$. Now, since we have assumed that $\phi \pi\left(E^{\prime}\right) \cap \gamma\left[\pi\left(E^{\prime}\right)\right]^{-1}$ is empty, we have

$$
\begin{aligned}
\left(q^{n}-1\right) /(q-1) & =\left|E^{\prime}\right| \geqq\left|\phi \pi\left(E^{\prime}\right) \cup \gamma\left[\pi\left(E^{\prime}\right)\right]^{-1}\right| \\
& =\left|\phi \pi\left(E^{\prime}\right)\right|+\left|\gamma\left[\pi\left(E^{\prime}\right)\right]^{-1}\right| \geqq q^{n-1}+q^{n-1}=2 q^{n-1} .
\end{aligned}
$$


Thus $q^{n-1}(q-2) \leqq-1$, which is impossible since $q \geqq 2$. Consequently, $\phi \pi\left(E^{\prime}\right) \cap \gamma\left[\pi\left(E^{\prime}\right)\right]^{-1}$ contains some element, $\varepsilon$ say. Thus $(\phi, \varepsilon) \in \pi\left(E^{\prime}\right)$ and $(\varepsilon, \gamma) \in \pi\left(E^{\prime}\right)$. Since $\pi\left(E^{\prime}\right)$ is transitive, it follows that $(\phi, \gamma) \in \pi\left(E^{\prime}\right)$.

We now have:

Theorem 3.5. Let $\mathrm{V}$ be an $n$-dimensional vector space over a finite field $F$. Let $\mathrm{Sing}_{n}$ denote the semigroup of singular endomorphisms of $\mathbf{V}$ and let $P F_{n-1}$ be the set of elements of Sing $_{n}$ with rank $n-1$. Then there exists a subset $E^{\prime}$ of the idempotents of $P F_{n-1}$ such that $E^{\prime}$ is a sparse cover for $P F_{n-1}$ and $E^{\prime}$ generates Sing $_{n}$. Further, any sparse cover for $P F_{n-1}$ generates Sing .

Proof. This is immediate from Lemma 2.2, Theorem 3.1 and Lemma 3.4.

(If $F$ is an arbitrary field, the above theorem no longer holds. A counter-example to a generalisation of Theorem 3.5 may be found in [2], as may a proof of the following weaker result.

Theorem 3.6. Let $\mathrm{V}$ be an n-dimensional vector space over an arbitrary field $F$. Let Sing ${ }_{n}$ denote the semigroup of singular endomorphisms of $\mathrm{V}$ and let $P F_{n-1}$ be the set of elements of $\operatorname{Sing}_{n}$ with rank $n-1$. Then there exists a subset $E^{\prime}$ of the idempotents of $P F_{n-1}$ such that $E^{\prime}$ is a sparse cover for $P F_{n-1}$ and $E^{\prime}$ generates Sing $g_{n}$ )

Corollary 3.7. Let $\mathbf{V}$ be an $n$-dimensional vector space over a finite field $F$ of order $q$. Let $\operatorname{Sing}_{n}$ be the semigroup of singular endomorphisms of $\mathrm{V}$ and let $E$ be the idempotents of Sing $_{n}$ of rank $n-1$. Then

$$
\min \left\{\left|E^{\prime}\right|: E^{\prime} \subseteq E,\left\langle E^{\prime}\right\rangle=\operatorname{Sing}_{n}\right\}=\left(q^{n}-1\right) /(q-1) .
$$

Proof. This is immediate from Lemma 2.4, Lemma 3.2 and Theorem 3.5.

\section{REFERENCES}

1. A. H. Clifford and G. B. Preston, The Algebraic Theory of Semigroups, vol. 1 (Math. Surveys of the American Mathematical Society no. 7, Providence, R.I., 1961).

2. R. J. H. Dawlings, Semigroups of Singular Endomorphisms of Vector Spaces, Ph.D. Thesis (University of St. Andrews, 1980).

3. J. A. ERDös, On products of idempotent matrices, Glasgow Math. J. 8 (1966), 118-122.

4. J. M. Howie, The subsemigroup generated by the idempotents of a full transformation semigroup, J. London Math. Soc. 41 (1966), 707-716.

5. J. M. HowIE, An Introduction to Semigroup Theory (Academic Press, 1976). 13.

6. J. В. КІм, Idempotent generated Rees matrix semigroups, Kyungpook Math. J. 10 (1970), 7-

Bayero UniverstTy

P.M.B. 3011, Kano, Nigeria 